DOI: $10.15393 /$ j3.art.2020.7890

UDC 517.518, 517.589

FOZI M. DANNAN

\title{
GENERALIZED KANTOROVICH CONSTANT, A NEW FORMULATION AND PROPERTIES
}

\begin{abstract}
A hyperbolic formulation has been established for the generalized Kantorovich constant. This formulation, besides some new inequalities for hyperbolic functions, allow us to obtain new properties of generalized Kantorovich constant, as well as to give short proofs for known properties. A dual generalized Kantorovich constant has been defined.
\end{abstract}

Key words: generalized Kantorovich constant; hyperbolic inequalities; hyperbolic formulation for Kantorovich constant; a dual generalized Kantorovich constant

2010 Mathematical Subject Classification: 47A63, 26D05, 26007

1. Introduction. The Kantorovich inequality is named after Soviet economist, mathematician, and Nobel Prize winner Leonid Kantorovich (1912-1986) for the inequality he established in 1948 [8].

An equivalent to the Kantorovich inequality was first proved by P. Schweitzer in 1914 [13].

The Kantorovich inequality has many applications. It is used in convergence analysis, and it bounds the convergence rate of the Cauchy steepest descent.

A variety of statistical applications of The Kantorovich inequality has been given by Gulhan Alpargu [1] in her very good survey thesis that contains historical information.

In 1948 Kantorovich [8] established the following inequality:

$$
\sum_{i=1}^{n} x_{i} p_{i} \sum_{i=1}^{n} \frac{1}{x_{i}} p_{i} \leqslant \frac{(m+M)^{2}}{4 m M},
$$

(C) Petrozavodsk State University, 2020 
for any $0<m \leqslant x_{j} \leqslant M, p_{j} \geqslant 0, j=1,2, \ldots, n$ with $\sum_{j=1}^{n} p_{j}=1$.

This inequality has been generalized in several directions because of its importance, see [2], [11].

The constant function

$$
H(m, M)=\frac{(m+M)^{2}}{4 m M}, 0<m<M
$$

which always appears in those articles, becomes itself an important item for research and generalizations, see [3], [4], and it is known as the Kantorovich constant. The first extension of the Kantorovich constant was given by Furuta [4] as follows:

$$
K(m, M, p)=\frac{(p-1)^{p-1}}{p^{p}} \cdot \frac{\left(M^{p}-m^{p}\right)^{p}}{(M-m)\left(m M^{p}-M m^{p}\right)^{p-1}}
$$

for $M>m>0$ and $p>1$. Note that $K(m, M, 2)=H(m, M)$. Putting $h=M / m$ in (1), we obtain

$$
K=\frac{(p-1)^{p-1}}{p^{p}} \cdot \frac{\left(h^{p}-1\right)^{p}}{(h-1)\left(h^{p}-h\right)^{p-1}}
$$

for any $p>1$. The generalized Kantorovich constant is formulated by Furuta [8] as follows:

$$
K(h, p)=\frac{h^{p}-h}{(p-1)(h-1)} \cdot\left(\frac{p-1}{p} \cdot \frac{h^{p}-1}{h^{p}-h}\right)^{p}
$$

for $h>1$ and any $p>1$ or $p<0$. It is not necessary to consider $0<h<1$, because $K(h, p)=K(1 / h, p)$.

The generalized Kantorovich constant has been used in many applications, especially in relative operator entropies, see [5-7], [9].

Also, the Kantorovich constant plays a vital role in various improvements of the Young inequality and its reverse for the scalar case, as well as for matrix versions, see [10], [12], [14], [15].

In this article, we introduce a new formulation for the generalized Kantorovich constant. Based on this formulation, we show that most of the known properties of the Kantorovich constant, see [3], [4], can be proved easily; also new properties will be obtained.

In the second section, we give several properties of the basic Kantorovich constant $H(m, M)$. In section 3 we give some inequalities for 
hyperbolic functions that are used in this article. In section 4 we introduce the hyperbolic formulation for the Kantorovich constant. A dual generalized Kantorovich constant will be defined in section 5 .

2. Properties of the basic Kantorovich constant. Let us start with some properties of the basic Kantorovich constant that can be written as follows:

$$
H(m, M)=H(1, x)=\frac{(x+1)^{2}}{4 x}=H(x),
$$

where $x=M / m$.

Proposition 1. The following properties of $H(1, x)$ (or simply $H(x)$ defined by (3)) hold:

(i) $H(m+1, M+1) \leqslant H(m, M) \leqslant H(m(m+1), M(M+1))$.

(ii) $H(x+y) \leqslant \frac{1}{2}[H(2 x)+H(2 y)]$ (convexity).

(iii) $H(x y) \geqslant H(x) H(y)$ (superadditivity).

(iv) $H(m, M)$ is decreasing with respect to $m$ and increasing with respect to $M$.

Proof. (i) We need the following identities:

$$
u^{2}(u+v+1)-(u+2)^{2} v=\left(u^{2}-4 v\right)(u+1)
$$

and

$$
\left(u^{2}+u-2 v\right)^{2}-u^{2}(u+v+1)=\left(u^{2}-4 v\right)\left(u^{2}+u-v\right),
$$

that hold true for any positive numbers $u$ and $v$. Note that those identities are interesting by themselves.

Putting $u=m+M$ and $v=m M$, it is not hard to verify that

$$
\begin{aligned}
H(m, M) & =\frac{u^{2}}{4 v}, \\
H(m+1, M+1) & =\frac{(u+2)^{2}}{4(u+v+1)}, \\
H(m(m+1), M(M+1)) & =\frac{\left(u^{2}+u-2 v\right)^{2}}{4 v(u+v+1)} .
\end{aligned}
$$

Therefore, from (4), we derive

$$
\frac{u^{2}}{4 v}-\frac{(u+2)^{2}}{4(u+v+1)}=\frac{\left(u^{2}-4 v\right)(u+1)}{4 v(u+v+1)}
$$


and in terms of $H(\cdot, \cdot)$, we have

$$
H(m, M)-H(m+1, M+1)=\frac{\left(u^{2}-4 v\right)(u+1)}{4 v(u+v+1)} .
$$

Now, using the identity (5), we get

$$
\frac{\left(u^{2}+u-2 v\right)^{2}}{4(u+v+1)}-\frac{u^{2}}{4 v}=\frac{\left(u^{2}-4 v\right)\left(u^{2}+u-v\right)}{4 v(u+v+1)},
$$

and in terms of $H(\cdot, \cdot)$, we obtain

$$
H(m(m+1), M(M+1))-H(m, M)=\frac{\left(u^{2}-4 v\right)\left(u^{2}+u-v\right)}{4 v(u+v+1)} .
$$

Since $u^{2}-4 v>0$ and $u^{2}+u-v>0$, it follows from (6) and (7) that

$$
H(m(m+1), M(M+1))>H(m, M)
$$

and

$$
H(m, M)>H(m+1, M+1) .
$$

The proof is complete.

(ii) Here we use the identity

$$
y(x+y)(2 x+1)^{2}+x(x+y)(2 y+1)^{2}-(x-y)^{2}=4 x y(x+y+1)^{2}
$$

that leads to

$$
\frac{(x+y+1)^{2}}{(x+y)}=\frac{(2 x+1)^{2}}{4 x}+\frac{(2 y+1)^{2}}{4 y}-\frac{(x-y)^{2}}{4 x y(x+y)}
$$

or, equivalently,

$$
2 H(x+y)=H(2 x)+H(2 y)-\frac{(x-y)^{2}}{8 x y(x+y)} .
$$

Therefore,

$$
2 H(x+y) \leqslant H(2 x)+H(2 y) .
$$

(iii) The required inequality can be written as follows:

$$
\frac{(x y+1)^{2}}{4 x y} \geqslant \frac{(x+1)^{2}}{4 x} \cdot \frac{(y+1)^{2}}{4 y}
$$


This inequality is equivalent to

$$
2(x y+1) \geqslant(x+1)(y+1)
$$

or

$$
(x-1)(y-1) \geqslant 0 .
$$

This completes the proof.

Property (iv) is got directly if we notice that

$$
\frac{d H}{d m}=\frac{m^{2}-M^{2}}{4 M m^{2}}<0 \text { and } \frac{d H}{d M}=\frac{M^{2}-m^{2}}{4 m M^{2}}>0 .
$$

Remark 1. Direct computations lead to the following additional properties:

$$
\begin{aligned}
H\left(x^{2}\right) & =[2 H(x)-1]^{2}, \\
H(x)+H(y) & =1+\sqrt{H(x y) H(x / y)}, \\
H(x)-H(y) & =\sqrt{[H(x y)-1][H(x / y)-1]}, \\
\sqrt{H(x)} & +\sqrt{H(x)-1}=\sqrt{x},
\end{aligned}
$$

where $H(x)=H(1, x)=(x+1)^{2} / 4 x$.

3. Inequalities for hyperbolic functions. In this section, we introduce some inequalities for hyperbolic functions that are interesting by themselves and necessary for the proofs in this article.

Lemma 1. For $x \geqslant y>0$, the following inequalities hold:

$$
\begin{gathered}
\frac{x}{y} \exp (y-x) \leqslant \frac{\sinh x}{\sinh y} \leqslant \frac{x}{y} \exp (x-y), \\
\frac{\cosh x}{\cosh y} \leqslant \frac{x}{y} \exp (x-y) .
\end{gathered}
$$

Proof. For the proof of the right-hand side of inequality (8), it is sufficient to show that the function $f(x)=\frac{\sinh x}{x e^{x}}=\frac{e^{2 x}-1}{2 x e^{2 x}}$ is decreasing. In fact, this is so, because

$$
f^{\prime}(x)=\frac{2 x+1-e^{2 x}}{2 x^{2} e^{2 x}}<0 \text { for } x \in R .
$$


Note that $f^{*}(x)=\frac{e^{x} \sinh x}{x}=\frac{e^{2 x}-1}{2 x}$ is an increasing function for $x \in R$, because

$$
f^{\prime *}(x)=\frac{2 x \exp (2 x)-\exp (2 x)+1}{4 x^{2}}>0 .
$$

The left-hand side of inequality (8) follows from this immediately. because

Similarly, since $g(x)=\frac{\cosh x}{x e^{x}}=\frac{\exp (2 x)+1}{2 x \exp x}$ is a decreasing function,

$$
g^{\prime}(x)=-\frac{2 x \exp (2 x)+\exp (2 x)+1}{2 x^{2} e^{2 x}}<0 \text { for } x \in R,
$$

we conclude the validity of (9).

Lemma 2. For $x>y>0$, the following inequality holds:

$$
\frac{x}{y}(\operatorname{coth} x-1)+1<\operatorname{coth} y<\frac{x}{y} \operatorname{coth} x .
$$

Proof. Since

$$
\left(\frac{x \cosh x}{\sinh x}\right)^{\prime}=\frac{\sinh (2 x)-2 x}{2 \sinh ^{2} x}>0,
$$

we obtain for $x>y$ that

$$
\frac{x \cosh x}{\sinh x}>\frac{y \cosh y}{\sinh y} \text { or } \operatorname{coth} y<\frac{x}{y} \operatorname{coth} x .
$$

To prove the left-hand side of inequality (10), it is sufficient to prove that

$$
m(x)=x \operatorname{coth} x-x=\frac{2 x}{\exp (2 x)-1}
$$

is a decreasing function. We have

$$
m^{\prime}(x)=\frac{(1-2 x) \exp (2 x)-1}{(\exp (2 x)-1)^{2}}=\frac{t(x)}{(\exp (2 x)-1)^{2}}
$$

Since $t(0)=0$ and $t^{\prime}(x)=-(1+2 x) \exp (2 x)<0$, it follows that $t(x)<0$ and, consequently, $m^{\prime}(x)<0$. 
4. Main results. In this section, we introduce the hyperbolic formulation and some properties for the generalized Kantorovich constant.

Proposition 2. The generalized Kantorovich constant can be written in the hyperbolic form as follows:

$$
K(h, p)=F(\theta, p)=\frac{1}{\sinh \theta} \cdot \frac{u_{p}(\theta)}{u_{p-1}(\theta)} ; \quad u_{p}(\theta)=\left(\frac{\sinh p \theta}{p}\right)^{p},
$$

where $\theta>0$ and $p>1$ or $p<0$.

Proof. Rewrite the generalized Kantorovich constant in the form

$$
K(h, p)=\frac{1}{h-1}\left[\left(\frac{h^{p}-1}{p}\right)^{p} /\left(\frac{h^{p}-h}{p-1}\right)^{p-1}\right] .
$$

Now, putting $h=e^{2 \theta}$ in (2) and using the identities

$$
\begin{aligned}
h^{p}-1 & =2 e^{p \theta} \sinh p \theta, \quad h-1=2 e^{\theta} \sinh \theta, \\
h^{p}-h & =\left[2 e^{(p-1) \theta} \sinh (p-1) \theta\right] e^{2 \theta}=2 e^{(p+1) \theta} \sinh (p-1) \theta,
\end{aligned}
$$

we obtain the expression (3) by substitution in (12).

This formulation enables us to obtain new properties and to give simple proofs for most of the known properties collected in the works of Furuta and Fujii [3], [4]. In what follows, we need the following Lemma.

Lemma 3. The function $u_{p}(\theta)$ satisfies the following properties:

(a) $\sinh ^{r} \theta \cdot \exp [-r(p+q-1) \theta] \leqslant \frac{u_{p}(\theta)}{u_{q}(\theta)} \leqslant \sinh ^{r} \theta \cdot \exp [r(p+q-1) \theta]$, where $r=p-q$ and $p>q>0$;

(b) $u_{p}(\theta) u_{-p}(\theta)=1$;

(c) $\frac{d}{d p} u_{p}(\theta)=u_{p}(\theta) \ell_{p}(\theta)$, where $\ell_{p}(\theta)=\ln \frac{\sinh p \theta}{p}+p \theta \operatorname{coth} p \theta-1$.

Proof. It is not hard to check the validity of the properties (b) and (c). For the right-hand side of (a) we have, for $r=p-q$ and $p>q>0$ :

$$
\frac{u_{p}(\theta)}{u_{q}(\theta)}=\frac{(\sinh p \theta)^{p}}{p^{p}} \cdot\left(\frac{q}{\sinh q \theta}\right)^{q}=\left(\frac{\sinh p \theta}{p}\right)^{r}\left(\frac{q}{p}\right)^{q}\left(\frac{\sinh p \theta}{\sinh q \theta}\right)^{q} .
$$

Applying inequality (8) (where $x=p \theta, y=q \theta$ ), we obtain

$$
\frac{u_{p}(\theta)}{u_{q}(\theta)} \leqslant\left(\frac{\sinh p \theta}{p}\right)^{r}\left(\frac{q}{p}\right)^{q}\left(\frac{p}{q}\right)^{q}\left(e^{r \theta}\right)^{q}=e^{r \theta q}\left(\frac{\sinh p \theta}{p}\right)^{r} \leqslant
$$




$$
\leqslant e^{r \theta q}\left(e^{(p-1) \theta} \sinh \theta\right)^{r}=\sinh ^{r} \theta \cdot \exp [-r(p+q-1)] .
$$

The left-hand side of (a) can be proved similarly.

Proposition 3. The generalized Kantorovich constant satisfies the following properties:

(i) $K(h, p) K(h, p-1) \ldots K(h, p-r)=\frac{1}{\sinh ^{r+1} \theta} \frac{u_{p}(\theta)}{u_{p-r-1}(\theta)}$ for $p>r+1$ and $r$ is a positive integer;

(ii) $h^{-(p+q-2)} \leqslant \frac{K(h, p)}{K(h, q)} \leqslant h^{(p+q-2)}$, where $r=p-q$ and $p>q>1$;

(iii) $h^{-p+1} \leqslant K(h, p) \leqslant h^{p-1}$;

(iv) $K^{p}\left(h^{p}, \frac{1}{p}\right)=\frac{1}{K(h, p)}$;

(v) $K(h,-p)=K(h, p+1)$;

(vi) $K_{p}^{\prime}(h,-p)=-K_{p}^{\prime}(h, p+1)$;

(vii) $K(h, 1-p)=K(h, p)$;

(viii) $K(h, p)=K(p)$ is an increasing function for $p>1$ and decreasing for $p<0$;

(ix) $K(h, p)=F(\theta, p)=\max _{-1 \leqslant t \leqslant 1} \frac{\cosh p \theta+t \sinh p \theta}{(\cosh \theta+t \sinh \theta)^{p}}$ for $p>1$ or $p<0$ and $\theta>0$.

Proof. (i) From (11) we have

$$
\begin{aligned}
& K(h, p) K(h, p-1) \ldots K(h, p-r)= \\
& =\frac{1}{\sinh ^{r+1} \theta} \frac{u_{p}(\theta)}{u_{p-1}(\theta)} \frac{u_{p-1}(\theta)}{u_{p-2}(\theta)} \cdots \frac{u_{p-r}(\theta)}{u_{p-r-1}(\theta)}= \\
& =\frac{1}{\sinh ^{r+1} \theta} \frac{u_{p}(\theta)}{u_{p-r-1}(\theta)}
\end{aligned}
$$

(ii) From inequality (a) in Lemma 3 it follows that

$$
\sinh \theta \cdot \exp [-2(p-1) \theta] \leqslant \frac{u_{p}(\theta)}{u_{p-1}(\theta)} \leqslant \sinh \theta \cdot \exp [2(p-1) \theta] .
$$

Therefore,

$$
\frac{\sinh \theta \cdot e^{-2(p-1) \theta}}{\sinh \theta \cdot e^{2(q-1) \theta}} \leqslant \frac{K(h, p)}{K(h, q)} \leqslant \frac{\sinh \theta \cdot e^{2(p-1) \theta}}{\sinh \theta \cdot e^{-2(q-1) \theta}}
$$


or

$$
\exp [-2(p+q-2) \theta] \leqslant \frac{K(h, p)}{K(h, q)} \leqslant \exp [2(p+q-2) \theta] .
$$

(iii) This inequality follows directly from (13).

(iv) Applying the hyperbolic formulation for $K(h, p)$, we obtain

$$
\begin{gathered}
K\left(h^{p}, \frac{1}{p}\right)=\frac{1}{\sinh p \theta} \cdot \frac{\left(p \sinh \frac{\theta p}{p}\right)^{\frac{1}{p}}}{\left(\frac{\sinh \left[\left(\frac{1}{p}-1\right) \theta p\right]}{\left(\frac{1}{p}-1\right)}\right)^{\left(\frac{1}{p}-1\right)}}= \\
=\frac{1}{\sinh p \theta} \cdot \frac{(p \sinh \theta)^{\frac{1}{p}}}{\left(\frac{p \sinh [(p-1) \theta]}{(p-1)}\right)^{\left(\frac{1}{p}-1\right)}}
\end{gathered}
$$

or

$$
\begin{aligned}
K^{p}\left(h^{p}, \frac{1}{p}\right)=\frac{p \sinh \theta}{(\sinh p \theta)^{p}} \cdot\left(\frac{p \sinh [(p-1) \theta]}{(p-1)}\right)^{(p-1)}= \\
=\frac{u_{p-1}(\theta)}{u_{p}(\theta)} \cdot \sinh \theta=\frac{1}{K(h, p)} .
\end{aligned}
$$

(v) $K(h,-p)=\frac{1}{\sinh \theta} \cdot \frac{u_{-p}(\theta)}{u_{-p-1}(\theta)}=\frac{1}{\sinh \theta} \cdot \frac{u_{p+1}(\theta)}{u_{p}(\theta)}=K(h, p+1)$

(vi) $K_{p}^{\prime}(h, p)=\frac{1}{\sinh \theta} \frac{d}{d p}\left(\frac{u_{p}(\theta)}{u_{p-1}(\theta)}\right) \frac{1}{\sinh \theta} \frac{u_{p}^{\prime} u_{p-1} u_{p} u_{p-1}^{\prime}}{u_{p-1}^{2}}$.

Applying Lemma 3, we obtain

$$
K_{p}^{\prime}(h, p)=K(h, p)\left[\ell_{p}(\theta)-\ell_{p-1}(\theta)\right]
$$

where

$$
\ell_{p}(\theta)=\ln \frac{\sinh p \theta}{p}+p \theta \operatorname{coth} p \theta-1
$$

Therefore,

$$
\begin{aligned}
& K_{p}^{\prime}(h,-p)=K(h,-p) {\left[\ell_{-p}(\theta)-\ell_{-p-1}(\theta)\right]=} \\
&=K(h, p+1)\left[\ell_{p}(\theta)-\ell_{p+1}(\theta)\right]=-K_{p}^{\prime}(h, p+1) .
\end{aligned}
$$


(vii) $K(h, 1-p)=\frac{1}{\sinh \theta} \frac{u_{1-p}(\theta)}{u_{1-p-1}(\theta)}=\frac{1}{\sinh \theta} \cdot \frac{u_{p}(\theta)}{u_{p-1}(\theta)}$.

Here we used $u_{p}(\theta) u_{-p}(\theta)=1$.

(viii) From equation (14), it is sufficient to show that $\ell_{p}(\theta)$ is increasing for $p>1$ and decreasing for $p<0$. For this purpose, we obtain, by differentiating (15), that

$$
\frac{d \ell_{p}(\theta)}{d p}=\frac{2 p \theta \sinh p \theta \cosh p \theta-p^{2} \theta^{2}-\sinh ^{2} p \theta}{p \sinh ^{2} p \theta} .
$$

Consider the function

$$
g(x)=2 x \sinh x \cosh x-x^{2}-\sinh ^{2} x,(x=p \theta) .
$$

Since $g(0)=0$ and $g^{\prime}(x)=4 x \sinh ^{2} x>0$ for $x>0$ and $g^{\prime}(x)<0$ for $x<0$, it follows that $g(x)>0$ for all $x \neq 0$. Therefore, $\frac{d \ell_{p}(\theta)}{d p}>0$ for $p>1$ and $\frac{d \ell_{p}(\theta)}{d p}<0$ for $p<0$.

(ix) Let

$$
F(t)=\frac{\cosh p \theta+t \sinh p \theta}{(\cosh \theta+t \sinh \theta)^{p}}
$$

Then $F^{\prime}(t)=0$ whenever

$$
t(1-p) \sinh \theta \sinh p \theta+\sinh p \theta \cosh \theta-p \sinh \theta \cosh p \theta=0 .
$$

This equation has a unique solution $t=t_{0}$, where

$$
t_{0}=\frac{1}{p-1}\left(\frac{\cosh \theta}{\sinh \theta}-p \frac{\cosh p \theta}{\sinh p \theta}\right)
$$

For $p>1$, put $x=p \theta$ and $y=\theta$ in inequality (10) and obtain

$$
p \frac{\cosh \theta}{\sinh \theta}+1-p<\frac{\cosh \theta}{\sinh \theta}<p \frac{\cosh p \theta}{\sinh p \theta} .
$$

From (18) and (19) it follows that $-1<t_{0}<0$. Furthermore, we conclude from (17) that $F^{\prime}(t)>0$ for $t<t_{0}<0$ and $F^{\prime}(t)<0$ for $t>t_{0}>0$. Therefore, a maximum of $F(t)$ takes place at $t=t_{0}$, and it follows that $\max _{-1 \leqslant t \leqslant 1} F(t)=F\left(t_{0}\right)$. In order to complete the proof, we show that $F\left(t_{0}\right)=F(\theta, p)$. To simplify the calculations, we denote $\sinh p \theta$, 
$\cosh p \theta, \sinh \theta, \cosh \theta$ by $S_{p}, C_{p}, S, C$ respectively. Now, the substitution of $t_{0}=\frac{1}{p-1}\left(\frac{C}{S}-p \frac{C_{p}}{S_{p}}\right)$ in (16) gives

$$
\begin{gathered}
\frac{C_{p}+\frac{1}{p-1}\left(\frac{C}{S}-p \frac{C_{p}}{S_{p}}\right) S_{p}}{\left[C+\frac{1}{p-1}\left(\frac{C}{S}-p \frac{C_{p}}{S_{p}}\right) S\right]^{p}}=\frac{\frac{1}{S(p-1)}\left(C S_{p}-S C_{p}\right)}{\left(\frac{p}{p-1}\right)^{p}\left(C-C_{p} \frac{S}{S_{p}}\right)^{p}}= \\
=\frac{1}{S} \frac{1}{p-1}\left(\frac{p-1}{p}\right)^{p} S_{p}^{p} \frac{C S_{p}-S C_{p}}{\left(C S_{p}-S C_{p}\right)^{p}}= \\
=\frac{1}{S} \frac{(p-1)^{p-1}}{p^{p}} S_{p}^{p} \frac{1}{\left(C S_{p}-S C_{p}\right)^{p-1}}= \\
=\frac{1}{S} \frac{(p-1)^{p-1}}{p^{p}} S_{p}^{p} \frac{1}{\left(S_{p-1}\right)^{p-1}}=\frac{1}{\sinh \theta} \cdot \frac{u_{p}(\theta)}{u_{p-1}(\theta)}=F(\theta, p) .
\end{gathered}
$$

For $p<0$, put $p=-p^{*}$, where $p^{*}>0$. Then $F^{\prime}(t)=0$ for a unique value $t=t_{1}$ defined by

$$
-t\left(p^{*}+1\right) \sinh \theta \sinh p^{*} \theta-\sinh p^{*} \theta \cosh \theta+p^{*} \sinh \theta \cosh p^{*} \theta=0
$$

or by

$$
t_{1}=\frac{1}{\left(p^{*}+1\right)}\left(p^{*} \frac{\cosh p^{*} \theta}{\sinh p^{*} \theta}-\frac{\cosh \theta}{\sinh \theta}\right) .
$$

From Lemma 2, as in the case $p>1$, we conclude that $0<t_{1}<1$ and $\max _{-1 \leqslant t \leqslant 1} F(t)=F\left(t_{0}\right)=F(\theta, p)$.

Remark 2. Properties (v), (vi) and (viii) have been proved by Furuta [4] using another approach.

Corollary. It is useful to write down some particular expressions that follow from Propositions 2 and 3.

$$
\begin{gathered}
K(h, 1)=1, \quad K(h, 2)=\cosh ^{2} \theta, \\
K(h, p) K(h, p-1)=\frac{1}{\sinh ^{2} \theta} \frac{u_{p}}{u_{p-2}}, \\
K(h, p) K(h, p-1) \ldots K(h, 1)=\frac{u_{p}}{\sinh ^{p-1} \theta}, \\
K_{p}^{\prime}=K(h, p)(\ell(p)-\ell(p-1)) .
\end{gathered}
$$


5. Dual generalized Kantorovich constant. At the end of this article, we define a dual Kantorovich constant which might be of interest.

Definition 1. Let $p>1$. The dual Kantorovich constant is defined by

$$
F^{*}(\theta, p)=\frac{1}{\cosh \theta} \cdot \frac{v_{p}(\theta)}{v_{p-1}(\theta)},
$$

where

$$
v_{p}(\theta)=\left(\frac{\cosh p \theta}{p}\right)^{p} .
$$

Note that the dual Kantorovich constant can be written as follows:

$$
K^{*}(h, p)=\frac{h^{p}+h}{(p-1)(h+1)} \cdot\left(\frac{p-1}{p} \cdot \frac{h^{p}+1}{h^{p}+h}\right)^{p},
$$

where $h=e^{2 \theta}$.

One can prove some properties for $K^{*}(h, p)$, similar to those of $K(h, p)$. Furthermore, we raise the following question:

Is there any relation between $F^{*}(\theta, p)$ and $F(\theta, p)$ defined by (11)? The following proposition gives a partial answer.

Proposition 4. For $p>r \geqslant 1$, we have the following identity:

$$
F(\theta, p-r+1) F^{*}(\theta, p-r+1)=\frac{(p-r)^{p-r}}{(p-r+1)^{p-r+1}} F(2 \theta, p-r+1) .
$$

Proof. Note that

$$
u_{n}(\theta) v_{n}(\theta)=\left(\frac{\sinh n \theta}{n}\right)^{n}\left(\frac{\cosh n \theta}{n}\right)^{n}=\frac{1}{(2 n)^{n}} u_{n}(2 \theta)
$$

from (11) and (20), we have

$$
\begin{aligned}
& F(\theta, p-r+1) F^{*}(\theta, p-r+1)= \\
& =\frac{1}{\cosh \theta} \cdot \frac{v_{p-r+1}(\theta)}{v_{p-r}(\theta)} \frac{1}{\sinh \theta} \cdot \frac{u_{p-r+1}(\theta)}{u_{p-r}(\theta)}=\frac{1}{\sinh 2 \theta} \cdot \frac{v_{p-r+1}(\theta)}{v_{p-r}(\theta)} \cdot \frac{u_{p-r+1}(\theta)}{u_{p-r}(\theta)}= \\
& =\frac{2}{\sinh 2 \theta} \cdot \frac{u_{p-r+1}(2 \theta)}{u_{p-r}(2 \theta)} \cdot \frac{[2(p-r)]^{p-r}}{[2(p-r+1)]^{p-r+1}}=\frac{(p-r)^{p-r}}{(p-r+1)^{p-r+1}} F(2 \theta, p-r+1) .
\end{aligned}
$$


Remark 3. For the basic Kantorovich constant

$$
H(x)=\frac{(x+1)^{2}}{4 x},(x>1)
$$

and the basic dual Kantorovich constant

$$
K^{*}(x, 2)=H^{*}(x)=\frac{\left(x^{2}+1\right)^{2}}{4 x(x+1)^{2}}, \quad(x>1)
$$

we have

$$
H^{*}(x)<H(x)<H^{*}(x)+1 .
$$

This follows from the identity

$$
H(x)-H^{*}(x)=1-\frac{x}{(x+1)^{2}} .
$$

\section{References}

[1] Alpargu Gulhan. The Kantorovich inequality, with some extensions and with some statistical applications. Master of Science thesis, McGill University, 1996.

[2] Baksalary J. K., Puntanen S. Generalized matrix versions of the Cauchy-Schwarz and Kantorovich inequalities . Aequationes Math., 1991, vol. 41, pp. $103-110$.

[3] Fujii Jun I., Fujii M., Seo Y. Bounds for interpolational path of positive operators, Recent topics in operator inequalities. 2004, vol.1359, pp. $46-57$.

[4] Furuta T. Specht Ratio S(1) can be expressed by Kantorovich constant $K(p): S(1)=\exp \left[K^{\prime}(1)\right]$ and its applications . Math. Inequal. Appl., 2003, vol. 6, no. 3, pp. 521-530.

[5] Furuta T. Extensions of Holder-McCarthy and Kantorovich Inequalities and Their Applications. Proc. Japan Acad., 1997, 73, Ser. A, pp. $38-41$.

[6] Furuta T. Basic properties of the generalized Kantorovich constant $K(h, p)=\frac{h^{p}-h}{(p-1)(h-1)} \cdot\left(\frac{p-1}{p} \cdot \frac{h^{p}-1}{h^{p}-h}\right)^{p}$ and its applications. Acta Scientiarum Mathematicarum, January 2004, 70(1).

[7] Furuta T. Two reverse inequalities associated with Tsallis relative operator entropy via generalized Kantorovich constant and their applications. Linear Algebra and its Applications, 2006, vol. 412, pp. $526-537$. 
[8] Kantorovich L. V. Functional analysis and applied mathematics . Uspechi Mayh. Nauk, 1948, vol. 3, pp. 89-185.

[9] Kluza P., Niezgoda M. Inequalities for relative operator entropies. Electronic Journal of Linear Algebra, 2014, vol. 27, December, pp. $851-864$.

[10] Liao W., Wu J., Zhao J. New versions of reverse Young and Heinz mean inequalities with the Kantorovich constant. Taiwanese Journal of Mathematics, 2015, vol. 19, no. 2, April, pp. 467-479.

[11] Mond B., Pečarić J. A matrix version of Ky Fan Generalization of the Kantorovich inequality. Linear and Multilinear Algebra., 1994, vol. 36, pp. $217-221$.

[12] Nasiri L., Shkalikov A. A., Shakoori M. A note on reverses of the Young type inequalities via Kantorovich constant. Studia Scientiarum Mathematicarum Hungarica, 2018, vol. 55 (3), pp. 363-373.

[13] Schweitzer P. An inequality concerning the arithmetic mean. Math. es phys. lapok, 1914, vol. 23, pp. 257-261.

[14] Yang Changsen, Ren Yonghui, Refinements and reverses ofYoung type Inequalities. International Journal of Mathematical Analysis, 2018, vol. 12, no. 2, pp. 53-60.

[15] Zuo H., Shi G., Fujii M. Refined inequality with Kantorovich constant. Journal of Mathematical Inequalities, 2011, vol. 5, no. 4, pp. 551-556.

Received September 03, 2019.

In revised form, February 09, 2020.

Accepted February 12, 2020.

Published online February 21, 2020.

Department of Basic Sciences Arab International University P.O.Box 10409, Damascus, SYRIA

E-mail: fmdan@scs-net.org 